\title{
Mediación didáctica con canciones narrativas en la Educación y la cultura histórica del Caribe colombiano
}

\author{
Benjamín Rocco Carrillo \\ roccocarrillob@gmail.com \\ Institución Educativa San Antonio de Piojó, Atlántico \\ Jeffrey Cardozo Niebles \\ jecardozo92@hotmail.com \\ Institución Educativa San Antonio de Piojó, Atlántico
}

\section{Resumen}

El presente artículo es producto de investigación cuyo objetivo fue diseñar una propuesta didáctica, fundamentada desde las canciones narrativas para el reconocimiento de la cultura histórica caribeña en estudiantes de básica secundaria. Metodológicamente, se asumió una postura cualitativa desde los planteamientos de Martínez (2014). A partir del tipo de investigación propositiva y diseño de campo. (Arias, 2006; Hurtado, 2012). Entre las unidades de análisis estuvieron músicos, licenciados en música y referentes de calidad del Ministerio de Educación Nacional. Se aplicaron guiones de entrevistas semiestructuradas y el análisis de contenido, instrumentos validados mediante juicios de cuatro (4) expertos. Las teorías de sustento se exponen desde los planteamientos de Sánchez (2009), Cataño (2011), en lo relacionado a la cultura histórica, y Gardey y Pérez (2009), Olmeda (2003), Ceriani (2013), para las canciones narrativas. Se concluyó que las instituciones educativas carecen de procesos de inter y transversalidad curricular que promuevan la cultura histórica y los procesos de construcción de identidad local, regional y nacional. Razón por la cual, se resaltan las canciones narrativas como mediación didáctica en los propósitos de comunicar costumbres, describir hechos históricos, reconocer temporal y geográficamente un contexto, y contribuir en los procesos de la composición lírica y narrativa desde sus elementos.

\section{Palabras clave}

Canciones narrativas; cultura histórica; mediación didáctica; 


\title{
Didactic mediation with narrative songs in the Education and historical culture of the Colombian Caribbean
}

\begin{abstract}
This article is the product of research whose objective was to design a didactic proposal, based on narrative songs for the recognition of Caribbean historical culture in students of basic secondary school. Methodologically, a qualitative stance was assumed from the approaches of Martínez (2014). From the type of propositional research and field design. (Arias, 2006; Hurtado, 2012). Among the units of analysis were musicians, music graduates and quality references from the Ministry of National Education. Scripts for semi-structured interviews and content analysis were applied, instruments validated through the judgment of four (4) experts. Livelihood theories are exposed from the approaches of Sánchez (2009), Cataño (2011), in relation to historical culture, and Gardey and Pérez (2009), Olmeda (2003), Ceriani (2013), for narrative songs. It was concluded that educational institutions lack curricular inter and transversal processes that promote historical culture and the processes of construction of local, regional, and national identity. Reason why, narrative songs are highlighted as didactic mediation in the purposes of communicating customs, describing historical events, temporally and geographically recognizing a context, and contributing to the processes of lyrical and narrative composition from its elements.
\end{abstract}

\section{Keywords}

Narrative songs, historical culture, didactic mediation. 


\section{Mediação didática com canções narrativas na educação e cultura histórica do Caribe colombiano}

\section{Resumo}

Este artigo é o produto de uma pesquisa cujo objetivo foi elaborar uma proposta didática, baseada em canções narrativas para o reconhecimento da cultura histórica do Caribe em estudantes do ensino médio. Metodologicamente, assumiu-se uma postura qualitativa a partir das abordagens de Martínez (2014). Do tipo de pesquisa proposicional e desenho de campo. (Arias, 2006; Hurtado, 2012). Entre as unidades de análise estavam músicos, graduados em música e referentes de qualidade do Ministério da Educação Nacional. Foram aplicados roteiros para entrevistas semiestruturadas e análise de conteúdo, instrumentos validados por julgamento de quatro (4) especialistas. As teorias de subsistência são expostas a partir das abordagens de Sánchez (2009), Cataño (2011), em relação à cultura histórica, e Gardey e Pérez (2009), Olmeda (2003), Ceriani (2013), para canções narrativas . Concluiu-se que as instituições de ensino carecem de processos inter e transversais curriculares que promovam a cultura histórica e os processos de construção da identidade local, regional e nacional. Razão pela qual, as canções narrativas são destacadas como mediação didática para fins de comunicação de costumes, descrevendo eventos históricos, reconhecendo temporal e geograficamente um contexto e contribuindo para os processos de composição lírica e narrativa a partir de seus elementos.

\section{Palavras-chave}

Canções narrativas, cultura histórica, mediação didática. 


\section{Introducción}

Promover el reconocimiento de la cultura histórica, implica un proceso complejo, donde prevalece la responsabilidad social como ciudadanos comprometidos con una nación pluriétnica y multicultural. Son estos, hechos de interés que fomentan la tradición, el folclor, y la relación del hombre con su pasado, teniendo como factor determinante sus valores culturales e identitarios como proceso clave para ilustrar grandes acontecimientos que han dejado huellas en la historia de la Región Caribe y todo el territorio colombiano.

En las categorías de análisis relacionadas con la cultura histórica, se han publicado distintas investigaciones y producciones bibliográficas desde los ámbitos internacional, nacional y local, que fundamentan y dan sustento teórico al presente estudio, permitiendo así, identificar la problemática socioeducativa expuesta, e identificar los aportes relevantes de las investigaciones citadas al presente estudio.

Previamente, desde el contexto internacional, Vides (2014), realizó una investigación titulada "Música como estrategia facilitadora del proceso enseñanza-aprendizaje", con el objetivo de determinar la metodología que utilizan los maestros en la implementación de la música como estrategia facilitadora del proceso enseñanza-aprendizaje dentro del aula.

Metodológicamente, el estudio se desarrolló desde el enfoque epistemológico cualitativo, a través del cual, se obtuvieron los resultados pretendidos. Se recopiló información contando con la participación de informantes claves desde su contexto real, permitiendo así, el análisis de la información para generar cuerpos teóricos confiables.

Entre las conclusiones más relevantes, se plantea que los maestros de educación básica primaria, secundaria y media, poco utilizan la música como elemento facilitador del conocimiento dentro del aula. Las observaciones realizadas, permitieron evidenciar lo antes expuesto, lo que invita al docente a replantear su metodología de enseñanza y poner en práctica estrategias basadas en la música, que contribuyan al reconocimiento de la cultura. Entre los aportes más importantes que hace dicho estudio a la presente investigación, se destaca la importancia y el valor que tiene la música como mediación en el proceso de enseñanza y aprendizaje. Asimismo, brinda al docente alternativas novedosas para la 
selección y organización de los contenidos programáticos, evidenciando los desempeños esperados por parte de los educandos.

Desde el contexto local, Rangel, y otros (2004), emprendieron una investigación titulada "La enseñanza de la historia de América Latina en $9^{\circ}$ a través del género musical salsa", con el objetivo de aplicar una propuesta pedagógica, musical y cultural, utilizando las canciones del género salsa como una alternativa para desarrollar las clases de historia en las instituciones educativas del Departamento del Atlántico. El mencionado proyecto, se desarrolló desde la metodología de enfoque cualitativo, indagando de manera directa la problemática y los factores asociados a la apatía de los estudiantes hacia temáticas referidas a la historia, hechos de interés que facilitaron el cambio de estrategias para favorecer al aprendizaje significativo de los estudiantes en esta área del conocimiento.

Entre las conclusiones más relevantes, se devela la falta de interés de los estudiantes en el aprendizaje de la historia, entre otros factores, está asociada a las metodologías usadas por los docentes, lo que motivo al diseño e implementación de nuevas estrategias que conduzcan a la formación integral del estudiante y la construcción de aprendizajes significativos desde la enseñanza de las ciencias sociales.

Como complemento, desde el ámbito local, Soto (2016), desarrolló un estudio titulado “Cantares del subdesarrollo como arquetipos de la Canción Crónica: A propósito del último álbum de Rubén Blades", con el objetivo de analizar la identidad cultural de los diferentes pueblos de América Latina, desde el contexto de ciertas canciones del mencionado cantautor panameño. En el texto, se destaca la importancia de construir identidad recreando el imaginario de las personas, todo con la finalidad de transformar el contexto regional latinoamericano.

Esta investigación, se desarrolló desde la metodología de enfoque cualitativo. Se indagó las cualidades y características de los contextos socioculturales, afirmando que las tradiciones y formas de expresión de cada pueblo constituyen una mirada de comprender el mundo presente. Asimismo, resaltó que las canciones del cantautor Rubén Blades, pueden contextualizarse en cualquier ciudad latinoamericana, adentrando en el imaginario del ser latinoamericano, desarrollando el pensamiento crítico y creando identidad. Canciones como 
Buscando América y País Portátil, son crónicas que narran de manera directa las raíces ancladas en el pasado, mezcladas con las problemáticas actuales, las cuales van desde lo

Finalmente, cerrando con el contexto local, Soto y Rodelo (2018), en su artículo titulado: "El cuerpo como subversión del baile "arrebatao" del barranquillero con la música "salsa": signos interculturales de nuestra identidad caribeña", destacaron el valioso aporte que ha hecho el género musical salsa y sus exponentes más importantes a la cultura caribeña y barranquillera, como son las letras, compases y ritmos, y en general, el contexto narrativo de las canciones de salsa que tocan las fibras del barranquillero.

El artículo, propuso concientizar al lector sobre la identidad caribeña, despertar en él, un sentido crítico y de pertinencia social. Estrechando así, los lazos del tejido social en el que sea participe para la construcción de nuevas perspectivas de transformar su contexto personal y social. Según los autores mencionados, es necesario pensar desde la música, y más aún desde las canciones del género salsa, las cuales representan en su mayoría las actividades cotidianas y el comportamiento humano, permitiendo construir cultura y seguirla conservando, como proceso esencial en la formación del individuo.

\section{Aproximación al concepto de cultura y cultura histórica}

Las exigencias de un mundo globalizado, requieren una mirada crítica a la educación en su misión de formar seres socialmente competentes, que reconozcan su historia y su pasado como pilar indispensable en la construcción de la ciudadanía y en el fomento de la diversidad cultural. Es importante resaltar que el término cultura es polisémico y multidisciplinar, por lo que a continuación se presenta otra significación de cultura, tal como la muestra Mayor, cuando expone:

La cultura es el conjunto de elementos simbólicos, estéticos y significativos que forman la urdimbre de nuestra vida y le confieren unidad de sentido y propósito, de la cuna a la tumba. La cultura modula la forma en que ejercemos el ocio, la dimensión y amplitud que damos a la vida, los horizontes que le fijamos y la forma en que superamos lo cotidiano e inmediato para buscar valores trascendentes (Mayor, 1994, p.4).

En este propósito, la cultura es el compendio del modo de vida y de todo aquello que caracteriza a las distintas sociedades, tanto sus acciones, como el modo de pensamiento y de 
ver el universo desde que se nace hasta que se muere, es lo que hace a unos diferentes de los demás, ya que la forma de pensar y las perspectivas que se tiene del mundo y de las cosas, es lo que le da sentido y otorga una identidad propia a cada grupo social.

Ahora bien, la actual preocupación, radica en cómo los docentes hacen más comprensible la definición de cultura a través de su práctica de enseñanza. Por décadas, la cultura se ha resumido en un breve concepto textual como sinónimo del conjunto de costumbres y creencias, relegando todo el lenguaje que habla de un contexto histórico y actual de la vida misma, de la interacción consigo mismo, con los demás y con su entorno.

Asimismo, entidades internacionales como la Organización de las Naciones Unidas para la Educación, la Ciencia y la Cultura (UNESCO), citado por Molano (2007), abordan el concepto de cultura como “(...) los rasgos distintivos, espirituales, materiales y afectivos que caracterizan una sociedad o grupo social. Ella engloba, además de las artes y las letras, los modos de vida, los derechos fundamentales del ser humano, los sistemas de valores, creencias y tradiciones" (p.72). Es decir, la cultura es vista como el conjunto de características que forman la esencia del ser humano en todas sus dimensiones, y estudia al hombre como ser individual y social desde sus leyes, patrimonio, emociones o prácticas determinantes que los distinguen de otros grupos sociales.

A partir de las concepciones anteriores sobre el término cultura, en las siguientes líneas se esbozará la cultura histórica como parte de ella, y como otra de las categorías de análisis principales del presente estudio. Sobre este concepto, Sánchez (2009), señala que esta “(...) es un proceso dinámico de dialogo social, por el que se difunden, se negocian y se discuten interpretaciones del pasado. (...) abarca, por lo tanto, múltiples narrativas y distintos enfoques, que pugnan por imponerse socialmente (...)” (p.277). Significa entonces, que, analizar la cultura histórica, implica propiciar debates donde sus miembros interpreten y comprendan las causas, elementos y factores que fundamentan sus rasgos sociales, logrando de este modo una relación con su pasado y comprensión del mismo, para dar explicación a los acontecimientos del presente y posibilitar un imaginario de su futuro.

Siguiendo en esta misma línea, Cataño (2011), expresa que la cultura histórica, puede propiciar “ (...) la relación que la persona, tanto en lo individual como en lo colectivo, establece con el pasado contribuye a lograr una explicación de sí misma y del mundo que 
habita, es decir, aporta a la forma en que se moldea la existencia humana (...)” (p.232). En otras palabras, en la cultura histórica se articulan los factores que caracterizan a los pensamientos y prácticas de una persona o determinado grupo social. Por esta razón, en las escuelas surge la necesidad desde las ciencias sociales, de inspirar en los estudiantes un sentido crítico que les permita comprender las situaciones del pasado, sus causas y consecuencias, para poder entender y dominar en mayor medida el contexto actual en el que viven.

Del anterior planteamiento, se deduce que no es posible adherirse al pasado de una manera fácil o con la simple descripción de hechos, sino que es necesario ilustrarlo en el presente a través de herramientas didácticas que motiven en los estudiantes el buen aprendizaje de las ciencias sociales. Es así, como los docentes de esta área, deben proponerse que la historia deje de ser vista como una asignatura aburrida, ya que esta puede ser contada a través de obras de teatro, películas, revistas culturales, visitas a lugares emblemáticos, memoria colectiva, canciones narrativas entre otros, donde sea representada y recreada de una forma significativa. De este modo, la 'cultura histórica' sintetiza la universidad, el museo, la escuela, la administración, los medios, y otras instituciones culturales como conjunto de lugares de la memoria colectiva, e integra las funciones de la enseñanza, del entretenimiento, de la legitimación, de la crítica, de la distracción, de la ilustración y de otras maneras de memorar, en la unidad global de la memoria histórica (Rüsen, 2009, p.3).

Del planteamiento anterior, se infiere que la cultura histórica se encarga de recopilar todos aquellos elementos que integran la esencia y la memoria colectiva e individual de las personas. Esta puede tratarse desde instituciones culturales, hasta lugares emblemáticos que propicien el diálogo y tertulias donde los individuos suelan reencontrarse con su pasado; propiciando un bagaje de aprendizajes y conocimientos concernientes a la naturaleza de los grupos humanos.

\section{Canciones narrativas como mediación didáctica en el proceso de enseñanza y aprendizaje}

Para la comprensión y análisis de la categoría relacionada con la música como mediación didáctica, es oportuno identificar conceptualmente la palabra mediación. Etimológicamente, el término 'mediar' proviene del latín mediāre, y señala la relación entre dos entes, sujetos o 
términos que sugieren un proceso dialéctico o de razonamiento. Hecha la observación anterior, es preciso considerar que en el ámbito educativo este término tiene otra connotación, el cual integra la acción docente con las interacciones pedagógicas que se pueden provocar a través del estudiante con su aprendizaje, el estudiante con sus pares, y el estudiante con su contexto, lo que conduce a la generación de experiencias de aprendizaje y al desarrollo integral del ser.

Desde la concepción de Escobar (2011), la mediación es considerada como uno de los principales dispositivos de aprendizaje y desarrollo emocional de las personas cuando afirma “(...) los procesos mentales superiores en los seres humanos son mediados por herramientas poderosas como el lenguaje, el mundo simbólico y el manejo de códigos” (p.59). En otras palabras, todo proceso de mediación requiere la intervención de cada una de las dimensiones que inciden en el desarrollo humano. Estas, están estrechamente relacionados con el contexto, la cultura, las relaciones sociales y afectivas, y la gestión del conocimiento, de tal manera que se puedan reestructurar o modificar algunos esquemas mentales ya establecidos. En síntesis, la mediación desde el contexto educativo, provoca la interacción entre las acciones del docente y estudiantes con el lenguaje, las expresiones afectivas, simbólicas, lingüísticas, entre otros; contribuyendo de forma positiva a las necesidades educativas, sociales y emocionales que aportan a la formación integral del ser humano. Hechas las consideraciones anteriores, y tomando el campo musical como base fundamental de mediación en el presente estudio, aplicado a la enseñanza de las ciencias sociales, surge la necesidad de exponer las teorías pedagógicas y didácticas que dan sustento a los procesos formativos en esta área.

Al respecto, la música es una manifestación artística y área de conocimiento dentro del campo educativo, que se enseña y se aprende de una manera vivencial a través de las estrategias didácticas creativas, intelectuales, emocionales y afectivas, las cuales pueden “(...) contribuir al desarrollo de la imaginación de los alumnos, de su creatividad original, pero para ello debe convertirse en un medio que estimule dicha imaginación y no en un mero artificio técnico que ahogue la inspiración personal” (Ruiz, 2011, p. 20).

Como se observa claramente, la educación musical juega un rol significativo en la formación integral del individuo. Sus aportes, permiten integrar otras áreas del saber, y coadyuvan a desarrollar funciones cognitivas en la solución de situaciones cotidianas, tal como postulan 
Bernal y Calvo (2004), "La educación musical desarrolla capacidades de observación, análisis y apreciación del hecho sonoro, lo que conduce a formular criterios de valoración, desarrollar preferencias y procedimientos.” (p. 26). En efecto, cabe resaltar que en la música se manifiestan valores, emociones e historias reales del diario vivir, por lo que, desde las distintas áreas del conocimiento, y en especial el área de sociales, ayuda tanto a docentes como estudiantes a implementar herramientas que generen espacios de aprendizajes enriquecedores.

Por lo anterior, la educación musical más que analizada desde la dimensión cognitiva y evaluada de manera cuantitativa, debe ser valorada de una forma cualitativa, flexible y liberadora, donde, intervienen otros componentes como la dimensión antropológica, la dimensión ética y estética; en la cual, se recopilan elementos propios de la música y su relación con la cotidianidad, debido a esto se justifica que:

(...) la música sigue siendo un acto singular, bello, estético, pero también es un acto social, uno que permite la solidaridad de los hombres y mujeres, que puede permitir además la posibilidad del diálogo entre ellos, y en este mismo sentido el de su proceso permanente de emancipación, en el que se liberan en comunión (Vargas, 2013, p. 60).

Cabe demostrar entonces, que la música además de ser un arte que auxilia procesos educativos, es un elemento que integra y culturiza a los individuos, generando en ellos hábitos y pautas de convivencia, la cual les permite expresar virtudes y sentimientos fraternos, adquiriendo así un sentido de liberación social. Por todo lo anterior, esta manifestación artística no se limita al uso de la voz y al simple manejo de instrumentos, sino que se extiende también al estudio del hombre como ser social en todas sus dimensiones, lo que conlleva a comprender la relación que existe entre la música y las ciencias sociales, tal como lo considera Lavín (2007), cuando afirma que “(...) las manifestaciones musicales van unidas a las condiciones culturales, económicas, sociales e históricas de algún espacio social. Para poder comprender un tipo de música concreto es necesario situarlo dentro del contexto cultural en el que ha sido creado (...)" (p.1).

Como puede observarse, no se deben desligar las expresiones musicales del contexto sociocultural donde son fundadas. La música se compone a partir de los procesos comunicativos que germinan de la propia cultura, razón por la cual, la educación musical se 
considera un campo flexible, capaz de promover dinámicas de impacto educativo, artístico y sociocultural.

\section{Interpretación del contexto de las canciones narrativas}

Atendiendo a las consideraciones anteriores, referentes a la música como mediación didáctica en el proceso de enseñanza y aprendizaje de las ciencias sociales, a continuación, se detallará la fundamentación teórica del término canción como categoría de análisis de esta investigación. En primer lugar, se presenta la canción desde el punto de vista coloquial como narraciones de historias y manifestación de sentimientos desde el campo musical. En efecto, Castello, dice:

Una canción es una composición musical para la voz humana (comúnmente acompañada por otros instrumentos musicales) y con letra. Típicamente es para un solo vocalista, aunque puede también ser para un dueto, trío, o para más voces (música coral). Las palabras de las canciones son tradicionalmente de versificación poética, aunque pueden ser versos religiosos de libre prosa. Las canciones pueden ser ampliamente divididas de muchas maneras distintas, dependiendo del criterio usado (Castello, 2009, p.15).

Del anterior planteamiento, se deduce que las canciones son poemas cuya voz humana interpreta de forma armónica con instrumentos musicales. Dicha interpretación, surge por lo general de la inspiración, inteligencia y experiencias vividas del compositor. La canción, es tradicionalmente interpretada por una sola persona a la que se le llama "vocalista", y esta puede ser acompañada por otras voces a las que se les llama “coro". Actualmente, son divididas por géneros musicales, estos a su vez, se clasifican dependiendo de los instrumentos con los que se armonicen las letras y a la interpretación del vocalista. Entre otros aspectos, las canciones también contribuyen a potencializar la memoria y la concentración para extender el vocabulario de quienes aprenden a través de ellas.

La canción es esencialmente un género literario, puede definirse como un texto elaborado bajo diversos criterios rítmicos y lingüísticos que lo particularizan y facultan para ser cantado. Así pues, a lo largo del tiempo, generalizada la práctica, no sabemos si por pulsión cultural o como efecto de una red de sentido, fueron añadiéndose elementos, texto y melodía que la hicieron más compleja y definieron paulatinamente los rasgos que hasta nuestros días 
caracterizan a la canción como un fenómeno artístico y cultural que vincula literatura y música (Vargas, 2019, p.20).

En relación con lo anterior, las canciones han sufrido diferentes cambios a lo largo de la historia, su evolución se ha visto ligada a la trasformación de la sociedad. Inició como un texto básico adornado de patrones musicales, al cual, con el pasar del tiempo se le fueron añadiendo valores sociales, políticos y culturales, llegando a considerarse en la actualidad como una composición musical literaria bastante completa, la cual, abarca de manera magistral todas las problemáticas del mundo actual. En otras palabras, las canciones forjan en el estudiante el fortalecimiento activo y armónico de habilidades auditivas, comunicativas, sensoriales y motoras que permiten un desarrollo multidimensional del mismo. De estas, se puede aprender según su contenido y su lenguaje en la medida que el estudiante indaga sobre los elementos, la estructura, el contenido y el contexto de la canción, que el compositor desea comunicar. Por tal motivo, la utilización de canciones en el aula, se han convertido en una herramienta de vanguardia que genera un ambiente propicio para el desarrollo de las clases. Las historias que narran las canciones, logran centrar mucho más la atención de los estudiantes, dándole significación al evento pedagógico, superando la precaria didáctica del dictado de una clase tradicional.

De acuerdo con los razonamientos preliminares, en el presente discurso, se exponen las canciones narrativas como una de las categorías que sustentan teóricamente esta investigación. En primer lugar, la canción narrativa se entiende popularmente como el tipo de composición musical cuyo contenido relata un sinnúmero de historias y crónicas, en su gran mayoría reales, transportando al oyente a un periodo histórico. En lo que respecta, Ceriani (2013), dice que “(...) en la temática de estas canciones podemos encontrar la narración de anécdotas y grandes acontecimientos que ocurrieron a lo largo de la historia en un determinado contexto" (p.5). Como se observa, una canción es una narrativa, caracterizada por elementos distintivos como: narrador (cantante), personajes, entorno, periodo histórico, contexto, hechos, y, en la mayoría de los casos, enseñanzas. Por otra parte, Milá (1869), plantea que la canción narrativa popular es un 
“(...) poema narrativo generalmente de breves dimensiones, compuesto para el pueblo y por el pueblo, y que se trasmite por medio de la tradición oral. La canción narrativa popular se divide según los asuntos en religiosa, heroica, romancesca, fantástica y doméstica" (p.193).

En este propósito, se entiende, además, que la canción narrativa popular habitualmente presenta su contenido de un modo no tan extenso, teniendo de manera intrínseca entre sus letras, particularidades esenciales e importantes de un pueblo; este tipo de canciones se transfieren oralmente de generación en generación. Un ejemplo de esto, es la canción "La Rebelión”, interpretada por Álvaro Arroyo González, conocido como "Joe Arroyo”, canción insignia de su repertorio musical, que narra un acontecimiento sucedido en la Región Caribe durante el siglo XVII, el cual transporta y contextualiza al oyente al periodo de la esclavitud en Colombia, ayudándolo entender además de su contenido, la historia de manera significativa.

Cabe agregar, según Lacoba (2011), que “(...) a través de la canción narrativa se nos cuentan historias, ya desde el primer verso de la canción la declaración de intenciones es clara. Algunas canciones narrativas no nos van a contar grandes historias, sino pequeñas anécdotas (...)" (p.1). Es decir, que es importante resaltar que una canción no ilustrará por completo una amplia historia debido a su duración, esta solo resaltará a manera de resumen los datos más relevantes de un hecho histórico, ya que no se puede narrar mucho en unas pocas estrofas.

En el ámbito educativo, las canciones narrativas no resolverán del todo una clase, estas serán una guía que estimulará el aprendizaje de los estudiantes, ayudándolos a comprender de manera más sencilla y eficaz las temáticas expuestas en el aula, sin embargo, la narración es un recurso indispensable en la canción, pues presenta de diferentes formas, sea como un relato, anécdota, un cuento o historia que permite darle movimiento, acción y vida a lo que se cuenta. Ella se desarrolla principalmente en los versos, mientras que el estribillo aparece un resumen de la historia, una moraleja. Si bien es posible pensar una canción sin narración, ella sería un monologo, un diálogo o una descripción. La narración está muy íntimamente 
relacionada con la canción, por lo que una canción sin narración es una canción sin historia, sin situaciones, sin movimiento o sin sucesión de hechos (Rothman, 2015, p.2).

Con relación al planteamiento preliminar, la canción narrativa es definida por Gómez y Pedrosa (2014), como “Canción extensa y compleja que desarrolla un argumento completo, con la correspondiente exposición, nudo y desenlace. En la tradición hispánica, cuando [en] un canto narrativo (...) su esquema es estrófico, constituye una canción narrativa propiamente dicha” (p.20). Significa que la canción narrativa se presenta de una manera mucho más amplia, ya que su contenido comprende un preámbulo, un desarrollo y, finalmente, una conclusión, con la finalidad de que el oyente entienda de manera secuencial lo que la canción expresa en su letra.

Entre las principales características de la canción narrativa, se destacan la forma de versos y los esquemas estrotíficos, tipologías que ayudan a utilizar las canciones narrativas como mediación para el reconocimiento de la cultura histórica, pues existen muchas canciones con estos caracteres que aportan a dicha cultura histórica, asimilándola y entendiéndola de una manera secuencial. En este sentido, para Rothman (2015), las canciones narrativas:

“(...) dejan múltiples enseñanzas, abordando temáticas que van desde lo político, pasando por lo cultural y geográfico, el estudiante al escuchar una canción de corte narrativo, enriquecerá su conocimiento y logrará comprender de manera más sencilla y eficaz las temáticas" (p.5).

Con relación a este postulado, un claro ejemplo de ello se puede evidenciar en las canciones del género vallenato, las cuales, se han constituido como ícono y patrimonio cultural de la Región Caribe colombiana, de la cual Escamilla y Morales consideran:

La canción narrativa en el vallenato parte de la incidencia en la recreación de algunos imaginarios culturales propios de la Región Caribe colombiana. Varios de esos imaginarios tienen que ver con las relaciones sentimentales de pareja, las cuales son presentadas en las canciones desde la perspectiva exclusivamente masculina de los compositores. También muestran una concepción particular de la felicidad, la amistad, la parranda, las costumbres ancestrales, sus creencias religiosas y los valores culturales y humanos de la región. Esta representación de la realidad que aparece en la canción vallenata no sólo tiene que ver con la recreación de un "mundo social" específico por parte de los compositores, sino también con el deseo o la voluntad de la gente del Caribe colombiano de ser percibida como distinta (Escamilla y Morales 2005, p.27). 
En síntesis, las canciones narrativas son estrategias de mediación didáctica en la enseñanza de las ciencias sociales, pues estas, son un puente que conlleva a la ruta del saber histórico en el reconocimiento de la cultura y su contexto. En esta misma línea, también se pueden citar como ejemplo, los negros traídos de África a América en condiciones de esclavos durante la época colonial, periodo en el que estos individuos utilizaban la música para expresar sus sentimientos por las circunstancias y tratos a los que eran sometidos. Según lo anterior, una canción narrativa es una forma de expresión en donde el individuo manifiesta las diferentes situaciones que le ha tocado vivir, como también describe los momentos más relevantes que han marcado el devenir de su comunidad. Esta, puede plasmar un suceso para la posteridad, dejando grandes enseñanzas que logran servir como ejemplo para las generaciones venideras. Gracias a estas, se pueden evocar grandes acontecimientos del pasado, un claro ejemplo de ello, es una canción titulada "La Puerta de Alcalá", interpretada por la "Grande De Madrid", en la cual, se narran los principales hechos ocurridos en una de las cinco antiguas puertas reales que daban entrada a la capital española, la ciudad de Madrid. Entre los hechos destacados en la canción, está la batalla de Madrid y la dictadura franquista. Otra canción narrativa acorde a esta definición es "El Padre Antonio y su monaguillo Andrés” de "Rubén Blades", basado en el asesinato del arzobispo salvadoreño Óscar Arnulfo Romero, el 24 de marzo de 1980 en el marco del conflicto armado en la ciudad de San Salvador.

A lo largo de los planteamientos hechos, se logra evidenciar que la narración es parte fundamental de la canción, ya que, por medio de esta, el oyente contextualiza de forma profunda lo que cuenta dicha composición musical. La narración es aquella que le otorga relevancia y sentido a la canción. Asimismo, es importante destacar que, lo que identifica a una canción narrativa es el fundamento descriptivo de la misma, lo que quiere decir, que una canción sin narración, es una canción que carece de argumentos, es una canción plana, sin historia y sin sentido.

\section{Discusión y análisis de los resultados}

Al preguntarles sobre la importancia que tiene la música como mediación didáctica en la escuela y en la formación del educando, los informantes coinciden en resaltar las cualidades 
y atributos a través de los cuales, la música contribuye a la formación del estudiante, entre las afirmaciones más relevantes están:

"La música tiene la capacidad de conectar al educando con los conocimientos (...)"

“(...) es la expresión cultural de los pueblos, a esto le agregamos la frase; somos lo que escuchamos y si nos proponemos a escuchar buena música seremos personas cultas".

"(...) la música como didáctica se conecta con la educación, que puede ser un enlace donde el intérprete se enfoca en la ciudad y su cultura".

"la música ayuda al educando a tener un proceso de enseñanza significativa al retener ciertas frases por medio del ritmo musical (...)".

En síntesis, los informantes coinciden en la funcionalidad de la música, resaltando que es de vital importancia en el ámbito educativo, mediante su narrativa explica de manera didáctica y veraz acontecimientos relevantes ocurridos a lo largo de la historia, incluso aborda distintas problemáticas de la vida social.

Después de lo anterior expuesto, es manifiesta la importancia de la música en el ámbito educativo, mediante su narración, porque explica de manera secuencial acontecimientos relevantes ocurridos a lo largo de la historia, incluso, aborda problemáticas de la vida social. Reafirmación señalada por el aporte de Pérez (2010), quien dice que "la integración musical es un campo de interacción social, cultural, científico, y de desarrollo humano, encaminado a promover las capacidades cognitivas de los (as) educandos, desde una educación de calidad. (p.11). En otras palabras, la música como mediación en la escuela, ayuda a formar grandes profesionales para la sociedad, en concordancia con las exigencias de la educación moderna, encaminada al mundo de la globalización. Seguidamente, al preguntarles sobre la función que tiene la música como mediación didáctica en el reconocimiento de la cultura histórica de la Región Caribe colombiana, emergen las siguientes afirmaciones:

"el Caribe colombiano como territorio de mezcla de ritmos necesita de la música como mediación didáctica para la conservación de sus raíces folclóricas, su reproducción, su perpetuación y reconocimiento como identidad inseparable".

"A través de una canción aprendemos y nos trasladamos a lugares sin viajar, por eso cuando escuchamos mencionar un lugar en una canción nos da la inquietud de averiguarla".

“(...) mediante la música podemos entrelazar la cultura y comprender las características primordiales del ser caribe".

“(...) ha enriquecido la cultura mediante las composiciones melodiosas.”. 
En conclusión, los informantes expresan que las canciones favorecen al conocimiento y despiertan la creatividad del estudiante en el proceso de entrelazar la cultura y comprender las características propias del ser caribe. Se concluye entonces, que la música es de gran utilidad al implementarla como mediación didáctica, debido a que su contenido narrativo ayuda inicialmente al desarrollo de habilidades lingüísticas, producción de textos y composición lírica, así como también al reconocimiento cultural e histórico de cualquier contexto geográfico.

En efecto, tales hallazgos se pueden relacionar con los postulados de Fernández (2011), quien establece que "la música y las ciencias sociales cuentan con un poderoso status, unidas poseen la capacidad de dirigir al alumno hacia estrategias y conocimientos de manera exitosa, su articulación logra facilitar aprendizajes que permiten aprender a aprender asertivamente” (p.35). En otras palabras, la música es una herramienta facilitadora de conocimientos, la cual, al ser unificada con las ciencias sociales, le permiten al joven analizar y comprender las temáticas de manera eficaz, y desde su contenido narrativo, permite la comprensión de temas afines con la geografía de cualquier región o contexto.

Al contrastar tal información con lo expuesto por Aguilera (2005), quien considera que "las canciones permiten la concientización, relación e identidad del individuo con los elementos y valores socio-culturales, locales, regionales y nacionales" (p.7), se puede afirmar, que este tipo de canciones además de ser populares, son también tradicionales y desde la didáctica de las ciencias sociales, se presentan acordes a las necesidades del contexto. Estos temas musicales narran de manera recreativa la cultura e idiosincrasia de los distintos corregimientos, municipios, distritos, ciudades, departamentos y demás entornos geográficos de la Región Caribe. A partir de los anteriores puntos de vista, se puede sintetizar que las canciones narrativas son aquellas que entrelazan lo lírico con lo narrativo, generando de esta manera un contenido que gira en torno a una acción o suceso ocurrido en algún lugar o contexto determinado. En concordancia con lo expuesto, Sáenz (2006), señala que “(...) la función de las canciones narrativas es servir de apoyo para la comprensión del pasado, estimulando inicialmente las emociones de los alumnos (...)" (P.78). Es decir, las canciones narrativas son de valioso aporte en la enseñanza de las ciencias sociales, sobre todo en la 
asignatura de historia, ya que se pueden hallar y analizar letras, historias, anécdotas, y descripciones de un determinado entorno. Asimismo, las canciones narrativas poseen un contenido lírico descriptivo que instruye al estudiante y lo conduce hacia un aprendizaje significativo y autónomo.

Al revisar sobre los principales músicos, intérpretes o compositores que han contribuido con la cultura histórica de la Región Caribe, porque sus obras son mediaciones importantes en las aulas escolares, se mencionan los nombres de algunas obras musicales de éstos:

"Tres Perlas", "Mi Vieja Barranquilla”, "En Barranquilla me quedo", "El hombre Caimán”, "la Fantástica", "La invitación de Jorge Celedón", "En Barranquilla Me Quedo", "La Rebelión", "El sol”, "El pescador" y "Los sabores de mi Porro".

De acuerdo a lo anterior, los expertos coincidieron al mencionar varios artistas considerados como grandes iconos de la historia musical, haciendo referencia a aquellos intérpretes y artistas que han hecho aportes importantes a nuestra historia a través de la música, entre ellos; Joe Arroyo, Adolfo Pacheco, Lucho Bermúdez y Pacho Galán, resaltando que las letras de sus canciones contribuyen al reconocimiento de la cultura Caribe. En este sentido Luna (2009), expresa que “(...) la enseñanza de la cultura histórica a través de las canciones permite acercar las fuentes escritas y la tradición oral en el aprendizaje" (P.3). En otras palabras, las canciones narrativas brindan a los estudiantes la posibilidad de asimilar la cultura histórica de la región a la que pertenecen, debido a que estas ilustran de manera didáctica el contexto, haciendo énfasis en sus letras, desarrollando la imaginación y creatividad. Asimismo, es de vital importancia destacar que los compositores juegan un papel fundamental antes de su interpretación, pues son quienes transmiten emociones, sentimientos y distintas temáticas y argumentos a través de las letras.

\section{Conclusiones}

Desde el proceso de análisis de los resultados, se sustentan las siguientes conclusiones:

Es oportuno, promover el reconocimiento de la cultura histórica para la formación integral del estudiante, permitiéndole desarrollar su identidad social y cultural, desde la identificación de rasgos distintivos propios del contexto local, región y nacional, forjando en él mayor sentido de pertenencia de sus creencias, valores y tradiciones. No obstante, el estudio 
permitió identificar múltiples factores que inciden en el reconocimiento de la cultura histórica de la Región Caribe.

Desde el contexto nacional, el Ministerio de Educación colombiano ha otorgado referentes de calidad sustentados desde lineamientos, estándares de competencia, Derechos Básicos de Aprendizaje (DBA), y las mallas de aprendizaje, para facilitar de alguna manera el proceso de planeación, gestión y evaluación curricular, sin embargo, estas orientaciones son muy generales y descontextualizadas de acuerdo a las necesidades de formación y del contexto local y regional. De acuerdo a la información aportada, los expertos exponen la necesidad de incorporar la música en el ámbito escolar, afirmando que necesitamos más didáctica musical en el aula de clases, mediante canciones y variedad de herramientas que permitirán al estudiante adquirir sus aprendizajes de manera más sencilla e innovadora. Al respecto, Aguilera (2005), destaca la importancia "que tiene la música como estrategia para la motivación en la comprensión de los contenidos académicos; además, contribuye con la integración social y la adquisición de una conducta ambientalista para la conservación del entorno al proporcionar elementos geográficos, históricos, ecológicos, sociales y otros que identifican e integran a una comunidad, municipio, estado o nación” (P.7). En otras palabras, la música es y seguirá siendo la mediación didáctica más asertiva para facilitar estrategias de motivación en la adquisición y conservación de elementos históricos y culturales de una región.

A los docentes, se les recomienda integrar las canciones narrativas en los procesos didácticos, estas, despiertan el interés de los estudiantes, facilitando la comprensión de temáticas complejas o descontextualizadas que generan actitud apática y reacia por parte de los jóvenes. La música es de gran utilidad al utilizarla como mediación didáctica, debido a que su contenido narrativo ayuda inicialmente al desarrollo de habilidades lingüísticas, producción de textos y composición lírica, así como también, al reconocimiento cultural e histórico de cualquier contexto geográfico.

Finalmente, es necesario concluir con la funcionalidad de la música en el ámbito educativo, resaltando como las canciones narrativas desde su lírica y narrativa recrean de manera didáctica acontecimientos relevantes ocurridos a lo largo de la historia, incluso aborda distintas problemáticas de la vida social. Razón por la cual, es oportuno que se diseñen 
currículos apropiados y coherentes con el contexto socioeducativo, es aquí donde este tipo de canciones se genera estrategia para estimular el intelecto del estudiante, logrando la fácil compresión de diferentes temáticas relacionadas con el ámbito social y los distintos procesos históricos en concordancia con la exigencia de la actual sociedad, que encaminada hacia el mundo de la globalización, merece rescatar el valor por el respeto y la inclusión desde la cultura musical y narrativa de los pueblos. 


\section{Referencias Bibliográficas}

Aguilera, O. (2005). La canción como recurso didáctico, Centro de Investigación, Difusión y Educación de Benítez (CINDIEB). Universidad Nacional Experimental "Simón Rodríguez" (UNESR). El Pilar, Estado Sucre, Venezuela.

Bernal, L., Calvo, M. (2004). Didáctica de la música, la voz y sus recursos. Repertorio de canciones y melodías para la escuela. Málaga, España: Editorial Aljibe, Ediciones.

Castello, L. (2009). ¿Qué es una canción? En Cuentos literatura infantil y juvenil.

Escamilla, J. y Morales, E. (2005). La canción vallenata como acto discursivo. Puerto Colombia, Colombia: Editorial: Círculo de análisis del discurso-cadis. Universidad del Atlántico.

Escobar, N. (2011). La mediación del aprendizaje en la escuela. Acción Pedagógica, № 20 (pp.58-73). San Cristóbal, Venezuela: Universidad Pedagógica experimental Libertador. Recuperado de: file://C:/Users/ASUS-H110M-R/Downloads/DialnetLaMediacionDelAprendizajeEnLaEscuela-6222147\%20(2).pdf

Fernández, A. (2011). Proyecto de Investigación Educativa: La música y la motivación en las evaluaciones. Recuperado de: http://www.slideshare.net/ximoneta_/proyectodeinvestigacin-educativa-la-msica-y-lamotivacin-en-las-evaluaciones-7632102

Gómez, N. y Pedrosa, J. (2014). Las voces sin fronteras: Didáctica de la literatura oral y la literatura comparada. Almería, España: Editorial: Universidad De Almería.

Lacoba, J. (2011). Canciones Liricas y Narrativas. Enredando con la lengua.

Lavín, J. (2007). Notas para un estado del arte de los estudios sobre música desde las ciencias sociales. Ciudad de México, México: Universidad Autónoma Metropolitana Xochimilco. Recuperado de: http://www.academia.edu/9486788/Notas_para_un_estado_del_arte_de_los_estudios_sobre _m\%C3\%BAsica_desde_las_ciencias_sociales_LA_M\%C3\%9ASICA_Y_LO_SOCIAL 
Luna, M. (2009). Docencia de la historia la música como vínculo entre la historia oral y la cultura juvenil. X Congreso Nacional de Investigación Educativa. Veracruz, México, 10 págs.

Mayor, F. (1994). La nueva página. Barcelona, España: UNESCO/Galaxia Gutenberg/Círculo de Lectores.

216

Molano, O. (2007). Identidad cultural un concepto que evoluciona Revista Opera, núm. 7, mayo, 2007, pp. 69-84 Universidad Externado de Colombia Bogotá, Colombia

Pérez, M. (2010). "La música como discurso dialógico e interdisciplinario en las instituciones de educación superior”. Universidad del Atlántico Barranquilla, Colombia.

Rodelo, M. y Soto, J. (2018). El cuerpo como subversión del baile “arrebatao" del barranquillero con la música "salsa": signos interculturales de nuestra identidad caribeña. Capítulo 8. Lenguaje interculturalidad y decolonización en América Latina. Universidad Del Atlántico. Disponible en: https://www.researchgate.net/publication/334453957_Capitulo_8_El_cuerpo_como_subver sion_del_baile_arrebatao_del_barranquillero_con_la_musica_salsa_signos_interculturales_ de_nuestra_identidad_caribena

Rothman, A. (2015). ¿Cómo escribir canciones y componer música? Portal web Escribir Canciones

Rüsen, J. (2009). ¿Qué es la cultura histórica?: Reflexiones sobre una nueva manera de abordar la historia. Culturahistórica. Versión castellana inédita del texto original alemán en K. Füssmann, H.T.

Sáenz, M. (2006). Las Canciones infantiles ¿Un recurso para la enseñanza de la historia? Campo Abierto: Revista de educación, 25(2), 75-88. 
Sánchez, E. (2005). "Una Revisión a Partir del Estudio de la Interacción Profesor-Alumnos en el Aula". Fundación Infancia y Aprendizaje.

Soto-Molina, Jairo E. (2016). Cantares del subdesarrollo como arquetipos de la Canción Crónica: A propósito del último álbum de Rubén Blades. Revista de Ciencias de la Educación, Docencia, Investigación y Tecnologías de la Información CEDOTIC. Vol. 2. No.2. PP.142-162. Disponible en: http://investigaciones.uniatlantico.edu.co/revistas/index.php/CEDOTIC/article/view/1874

Vargas, I. (2013). Sistematización de una práctica de la pedagogía del oprimido: desde una experiencia en educación musical con los jóvenes de octavo grado del colegio república de Colombia (tesis de pregrado). Bogotá, Colombia: Universidad Pedagógica Nacional. Recuperado de: http://repositorio.pedagogica.edu.co/bitstream/handle/20.500.12209/1705/TE11123.pdf?seq uence $=1 \&$ is Allowed $=\mathrm{y}$

Vargas, Y. (2019). La canción como género literario. rhythmus, literatura musical.

Vides, A. (2014). "Música Como Estrategia Facilitadora del Proceso EnseñanzaAprendizaje". Ciudad de Guatemala, Guatemala: Universidad Rafael Landívar. 